\title{
Introduction to Neuroimaging Techniques in the Diagnosis of Brain Cancer Metastases
}

Before the advent of X-ray imaging, cases of metastatic brain lesions were considered rare; furthermore, the direct application of this diagnostic method revolutionary for that time did not allow to identify brain tumors on radiographs, with exception of defects in the skull bones indirectly indicative of their tumor origin (Oppenheim 1901). In the early twentieth century, air encephalography (1919) was put into practice, followed by cerebral angiography (1931), allowing to detect changes in the brain vasculature and identify tumor vessels by their contrast enhancement (Dandy 1919 and Monitz 1927). In 1936, the method of electroencephalography (EEG) discovered as early as in 1875 was first applied to determine locations of brain tumors.

The advent of computed tomography (CT) in the late 1970s of twentieth century for the first time made possible direct X-ray imaging of brain tumors. However, only the introduction of CT contrast agents provided an opportunity for detection and identification of metastases among other brain tumors (Konovalov and Kornienko 1985a, b; Hardy et al. 1990; Khanjanasthiti et al. 1989). New opportunities in the diagnosis of brain metastases of malignant tumors with various locations occurred with the advent of magnetic resonance imaging (MRI) (Sze et al. 1990; Kuhn et al. 1994; Akeson et al. 1995; Schellinger et al. 1999). The development of radioisotope diagnosis, in particular single-photon emission computed tomography (SPECT) and positron emission tomography (PET), expanded the scope of the resulting visual information about tumors in various organs and tissues of the body and estimates of metabolic disorders in the brain substance and allowed to identify metastatic lesions, including those in the brain (Kitajima et al. 2009; Abdelmalik et al. 2013). It should be noted that X-ray imaging and cerebral angiography were the first imaging techniques used in neuro-oncology (Arutyunov and Kornienko 1971).

Among a variety of diagnostic techniques currently in use for investigation of internal human tissues and body fluids, it is impossible to single out one that best displays all the information necessary for clinicians, characterizing brain metastases. According to most neuroradiologists, a comprehensive diagnosis is required to assess brain metastases, including cerebral angiography, CT, MRI, single-photon emission computed tomography, and positron emission tomography (Kornienko and Pronin 2010; Abdelmalik et al. 2013). These techniques do not duplicate each other, since they differ in their "specialization"; therefore, each of them is used as part of the diagnostic system to solve only those problems where it has an advantage over the others: one is used to identify the primary foci; the other one for the detection of metastatic lesions, evaluation of their structure, and relationship with surrounding tissues; and the third one to clarify tumor growth and blood supply patterns. In addition, they allow to study changes in tumor growth, carry out a postoperative monitoring of patients, and evaluate the effectiveness of cancer treatment. 